\title{
Simulation of non-linear ultrasound fields
}

\author{
Jensen, Jørgen Arendt; Fox, Paul D.; Wilhjelm, Jens E.; Taylor, Louise Kold
}

Published in:

IEEE Ultrasonics Symposium

Link to article, DOI:

10.1109/ULTSYM.2002.1192632

Publication date:

2002

Document Version

Publisher's PDF, also known as Version of record

Link back to DTU Orbit

Citation (APA):

Jensen, J. A., Fox, P. D., Wilhjelm, J. E., \& Taylor, L. K. (2002). Simulation of non-linear ultrasound fields. In IEEE Ultrasonics Symposium (Vol. 2, pp. 1733-1736). IEEE. https://doi.org/10.1109/ULTSYM.2002.1192632

\section{General rights}

Copyright and moral rights for the publications made accessible in the public portal are retained by the authors and/or other copyright owners and it is a condition of accessing publications that users recognise and abide by the legal requirements associated with these rights.

- Users may download and print one copy of any publication from the public portal for the purpose of private study or research.

- You may not further distribute the material or use it for any profit-making activity or commercial gain

- You may freely distribute the URL identifying the publication in the public portal

If you believe that this document breaches copyright please contact us providing details, and we will remove access to the work immediately and investigate your claim 


\title{
Simulation of non-linear ultrasound fields
}

\author{
Jørgen Arendt Jensen, Paul D. Fox, Jens E. Wilhjelm and Louise Kold Taylor \\ Center for Fast Ultrasound Imaging, Ørsted•DTU, Bldg. 348, \\ Technical University of Denmark, DK-2800 Kgs. Lyngby, Denmark
}

\begin{abstract}
An approach for simulating non-linear ultrasound imaging using Field II has been implemented using the operator splitting approach, where diffraction, attenuation, and non-linear propagation can be handled individually. The method uses the Earnshaw/Poisson solution to Burgers' equation for the non-linear propagation. The speed of sound is calculated from the instantaneous pressure of the pulse and the nonlinearity B/A parameter of the medium. The harmonic field is found by introducing a number of virtual planes in front of the aperture and then propagating the pulse using Burgers' solution between the planes.
\end{abstract}

Simulations on the acoustical axis of an array transducer were performed and compared to measurements made in a water tank. A $3 \mathrm{MHz}$ convex array transducer with a pitch of $0.53 \mathrm{~mm}$ and a height of $13 \mathrm{~mm}$ was used. The electronic focus was at $45 \mathrm{~mm}$ and 16 elements were used for emission. The emitted pressure was $1.4 \mathrm{MPa}$ measured $6 \mathrm{~mm}$ from the aperture by a Force Institute MH25-5 needle hydrophone in a water bath. The build-up of higher harmonics can here be predicted accurately up to the 5 th harmonic. The second harmonic is simulated with an accuracy of $\pm 2.6 \mathrm{~dB}$ and the third harmonic with $\pm 2 \mathrm{~dB}$ compared to the water bath measurements. Point spread functions (PSFs) were also calculated and measured. They all showed that the second and third harmonic PSFs are narrower than for the first harmonic, with a good resemblance between the measured and simulated PSFs.

The approach can also be extended to simulate non-linear ultrasound imaging in 3D using filters or pulse inversion for any kind of transducer, focusing, apodization, pulse emission and scattering phantom. This is done by first simulating the non-linear emitted field and assuming that the scattered field is weak and linear. The received signal is then the spatial impulse response in receive convolved with the emitted field at the given point.

\section{INTRODUCTION}

Modern medical ultrasound scanners employ the non-linear propagation of ultrasound to generate harmonic images. Such images have resulted in a higher contrast and a lower influence from side and gratings lobes especially for cardiac imag- ing. For the optimization of the imaging, it is important to understand the build-up of the non-linear fields and how different transducer parameters affect the non-linear generation. The studying of these effects is greatly facilitated by a simulation tool, where parameter studies can easily be made. In this paper a solution based on Field II [1] is investigated. The program makes it possible to study all kinds of transducer geometries, focusing and apodization schemes and general imaging can be implemented. The non-linear part of the program is based on the Earnshaw/Poisson solution to Burgers' equation for the non-linear propagation.

In solving the nonlinear differential equations, it can be an advantage to decouple the different effects (in this study diffraction, attenuation, and nonlinearity) and calculate each effect separately. The sum of the effects is then found by adding all effects at each integration step. Each integration step handles the influence of the effects over a limited spatial range based on the simulated acoustic field determined at the previous step. This method is referred to as the Operator Splitting Method [2, 3].

The purpose is here to expand Field II's applicability to also include the nonlinear effects, so that the generation of higher harmonics created by medical ultrasound array transducers can be studied. The approach will be based on the operator splitting method in a similar manner as Tavakkoli et al. (1998) and Remenieras et al. (2000), but using the spatial impulse response approach based on the Rayleigh integral together with the Earnshaw/Poisson solution to Burgers' Equation. Attenuation is handled by Field II as a filter on the spatial impulse responses. This paper expands upon the nonlinear propagation of virtual planes previously described in [4], and shows how point spread functions can be calculated by the program.

\section{PROPAGATION MODEL}

The Westervelt Equation [5] describes the nonlinear propagation of a sound wave. It is a second order approximation for thermoviscous fluids derived from expansions of equations of mass conservation, momentum conservation, entropy bal- 
ance, and thermodynamic state [6]. The equation reads:

$$
\left(\nabla^{2}-\frac{1}{c_{0}^{2}} \frac{\partial^{2}}{\partial t^{2}}\right) P+\frac{\delta}{c_{0}^{4}} \frac{\partial^{3} P}{\partial t^{3}}+\frac{\beta}{\rho_{0} c_{0}^{4}} \frac{\partial^{2} P^{2}}{\partial t^{2}}=0
$$

where $\nabla^{2}=\partial^{2} / \partial \mathbf{r}^{2}$ is the Laplacian operator, $P$ is the change in pressure relative to ambient pressure, and is a function of time, $t$, and spatial position, $\mathbf{r}$. The small-signal sound speed is represented by $c_{0}, \rho_{0}$ is the density of the medium, and $\delta$ is the diffusivity of sound describing attenuation in the medium. The first two terms in the parenthesis represent diffraction and linear propagation, and the third term describes thermoviscous losses. The nonlinear effect on wave propagation is governed by the last term on the left hand side. The parameter $\beta$ determines the nonlinearity. It is defined as $\beta=1+B / 2 A$, where $B / A$ is the ratio describing nonlinearities in media. Usually $\beta$ takes values in the range 6-10 for biological tissues [6].

To solve and thereby use (1) for the simulation using the operator splitting method, it will be appropriate to look closer at (1) and rewrite it. The loss term represents a quadratic dependence with frequency [6]. Since the attenuation (in $\mathrm{dB}$ ) shows a close to linear dependence with frequencies in the ultrasound region for most biological tissues [7], this term is not appropriate. For this reason, $\delta$ is set equal to zero at this point, and a frequency dependent attenuation is introduced later as a filter on the pressure waveform. The lossless version of (1) reads:

$$
\left(\frac{\partial^{2}}{\partial \mathbf{r}^{2}}-\frac{1}{c_{0}^{2}} \frac{\partial^{2}}{\partial t^{2}}\right) P+\frac{\beta}{\rho_{0} c_{0}^{4}} \frac{\partial^{2} P^{2}}{\partial t^{2}}=0
$$

At this point a retarded framework, where the observer follows the wave, is introduced. The new time variable is defined as $\tau=t-|\mathbf{r}| / c_{0}=t-r / c_{0}$, and the above equation can be transformed to:

$$
\frac{\partial P}{\partial \mathbf{r}}=\int \frac{c_{0}}{2} \frac{\partial^{2} P}{\partial \mathbf{r}^{2}} d \tau+\frac{\beta}{2 \rho_{0} c_{0}^{3}} \frac{\partial P^{2}}{\partial \tau} .
$$

By means of operator splitting [2,3], a numerical integration can be carried out by solving the equation for one effect at the time over a small integration step, $\Delta r$, and then adding the two effects. By transforming back to the original time axis $t=$ $\tau+|r| / c_{0}$, the contribution associated with the first becomes the lossless linear equation $\left(\partial^{2} / \partial r^{2}-1 / c_{0}^{2} \cdot \partial^{2} / \partial t^{2}\right) P=0$, which may be solved using the spatial impulse response $[8,9]$ implemented and used in Field II [10]. The second term however represents the lossless nonlinear Burger's equation $[11,6]$, which after differention of the squared pressure term may be written

$$
\frac{\partial P}{\partial r}+\frac{1}{c_{r}} \frac{\partial P}{\partial \tau}=0 \quad: \quad \frac{1}{c_{r}}=-\frac{\beta P}{\rho_{0} c_{0}^{3}}
$$

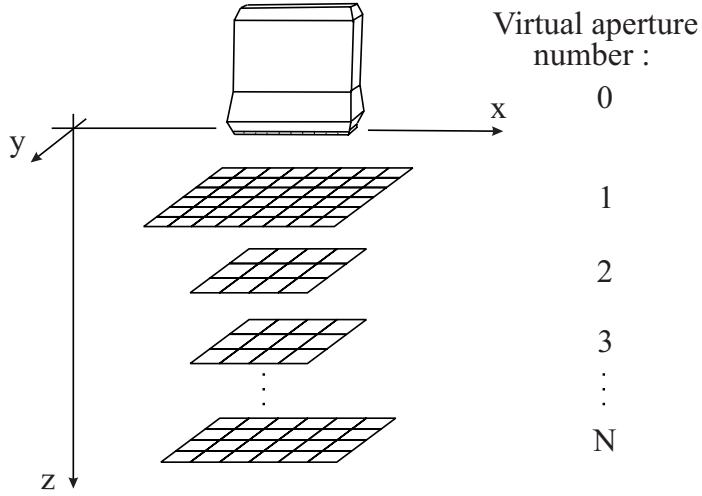

Figure 1: Virtual ultrasound planes for propagating the nonlinear field.

In the event that $1 / c_{r}$ may be considered constant, this equation has solution $P=P(r, \tau)$ which propagates spatially from $r$ to $r+\Delta r$ according to

$$
\begin{aligned}
P(r+\Delta r, \tau) & =P(r, \tau+\Delta \tau) \\
\Delta \tau & =-\Delta r / c_{r}=\Delta r \beta P(r, \tau) / \rho_{0} c_{0}^{3} .
\end{aligned}
$$

This is the Earnshaw/Poisson solution [6]. Hence, if we consider step sizes $\Delta r$ sufficiently small to approximate $c_{r}=$ $-\rho_{0} c_{0}^{3} / \beta P(r, \tau)$ as constant (i.e. $P$ constant), then the nonlinear component $P(r+\Delta r, \tau)$ may be computed simply as the time shifted field $P(r, \tau+\Delta \tau)$ at the previous position position $r$. Note here that since $\Delta \tau$ is a function of $P(r, \tau)$, the time shift is not constant as the wave propagates, and it is this mechanism which introduces the nonlinear distortions into the wavefront.

The simulation of the acoustic field generated due to the diffraction, attenuation, and nonlinearity is handled by following the simulation setup shown in Fig. 1. A number of successive steps are taken from the transducer and out into the field. Each propagation step evaluates the pressure waveforms on elements in a plane - a virtual aperture, which serves as input aperture for the next propagation step. The following steps are, thus, made:

1. Calculate the attenuated pulses at the center position of the elements in the next virtual plane.

2. Disturb the pulses to make non-linear propagation.

3. Set pulses as excitation for the next plane.

4. Repeat the procedure for the next virtual plane.

\section{SIMULATION AND EXPERIMENTAL SETUPS}

The ability to simulate the propagation of an acoustic field with the above described method is determined by comparing 


\begin{tabular}{lr} 
Center frequency, $f_{0}$ & $3 \mathrm{MHz}$ \\
No. of cycles & 5 \\
No. of elements & 16 \\
Pitch & $0.525 \mathrm{~mm}$ \\
Kerf & $40 \mu \mathrm{m}$ \\
Transverse length & $13 \mathrm{~mm}$ \\
Radius of curvature & $60 \mathrm{~mm}$ \\
Acoustical focus & $70 \mathrm{~mm}$ \\
Electronic focus & $45 \mathrm{~mm}$ \\
Distance between measurements & $6 \mathrm{~mm}$ \\
Number of measurements & 45 \\
No. of lines averaged & 8 \\
& \\
\multicolumn{1}{c}{ Table 1: Experimental parameters } \\
Lateral length, x & $20.5 \mathrm{~mm}$ \\
Transverse length, y & $15.5 \mathrm{~mm}$ \\
Element size, $\Delta x$ & $0.5 \mathrm{~mm}$ \\
Element size, $\Delta y$ & $0.5 \mathrm{~mm}$ \\
Distance between planes, $\Delta z$ & $6 \mathrm{~mm}$ \\
Density of medium, $\rho_{0}$ & $1000 \mathrm{~kg} / \mathrm{m}^{3}$ \\
Nonlinearity parameter, $\beta$ & 3.5 \\
Propagation step size, $\Delta r$ & $0.5 \mathrm{~mm}$ \\
Pressure at depth $6 \mathrm{~mm}, P_{0}$ & $1.4 \mathrm{MPa}$ \\
Speed of sound, $c_{0}$ & $1480 \mathrm{~m} / \mathrm{s}$
\end{tabular}

Table 2: Simulation parameters

the simulated data to data acquired from hydrophone measurements in a water tank. Table 1 and 2 lists the relevant parameters for both the measurements and the simulations. The measured waveforms were obtained by emitting a pulse with a $3 \mathrm{MHz}$ convex array probe on a B-K Medical 2102 scanner and LeCroy 9450 oscilloscope at a sampling frequency of 200 MHz coupled to a Force Institute MH25-5 needle hydrophone in a water bath. The $3 \mathrm{MHz}$ convex array transducer has an element width of $0.53 \mathrm{~mm}$ and a height of $13 \mathrm{~mm}$. The electronic focus was at $45 \mathrm{~mm}$, the elevation focus at $70 \mathrm{~mm}$, and 16 elements were used for emission.

Simulations on and off the acoustical axis of an array transducer were performed and compared to measurements made in a water tank.

\section{RESULTS}

An example of a simulated pulse and the corresponding spectrum is shown in Fig. 2. The solid line is the measured pulse and the dashed line is the simulated responses. It can be seen that the general shape of the pulse is predicted accurately, but the amplitude is slightly underestimated primarily due to the underestimation of the higher harmonics.

The resulting point spread functions are shown for in Fig. 3. For contour lines at $-6,-12,-18$ and $-24 \mathrm{~dB}$ the point
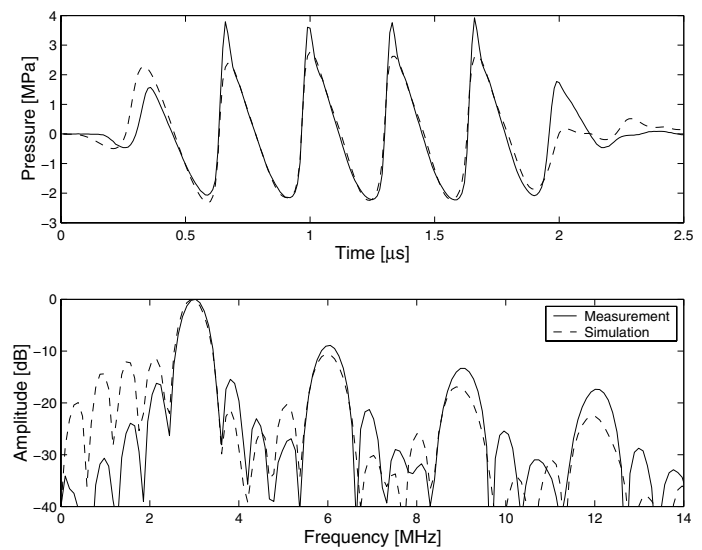

Figure 2: Example of simulated and measured pulse (top) and corresponding spectrum (bottom) at $z=24 \mathrm{~mm}$.

spread functions for the fundamental, second, and third harmonic are shown as a function of lateral position. The simulated data are shown on the top and the measured on the bottom. Roughly the same lateral extend of the fields are predicted by the simulation as found in the measurements. There is, however, more noise in the simulated response in the -24 $\mathrm{dB}$ contours and here the PSFs are not correctly predicted.

The build-up of the harmonic components are shown Fig. 4. On the left is shown the second harmonic relative to the first harmonic and in the right is shown the third harmonic relative to the first harmonic. The rise of the harmonics with depth is predicted by the simulation with an overall standard deviation of $2.6 \mathrm{~dB}$ for the second harmonic and $2 \mathrm{~dB}$ for the third harmonic compared to the measured data.

\section{$\mathrm{V}$ EXTENSION TO NON-LINEAR IMAGING SIMULATION}

The approach can also be extended to simulate non-linear ultrasound imaging in 3D using filters or pulse inversion for any kind of transducer, focusing, apodization, pulse emission and scattering phantom. This is done by first simulating the non-linear emitted field and assuming that the scattered field is weak and linear. The received signal is then the spatial impulse response in receive convolved with the emitted field at the given point. For linear array imaging, the emitted field is only calculated once, since it is the same for all image lines. The received signal is then found by moving the scatterers into the correct position compared to the emitted field. The non-linear emitted field at the different points is then calculated by finding the plane closets to the point and use this in the calculation, and then find the received signal by convolving with the spatial impulse response for the receiving aperture.

Tissue mimicking computer phantoms can then be made 

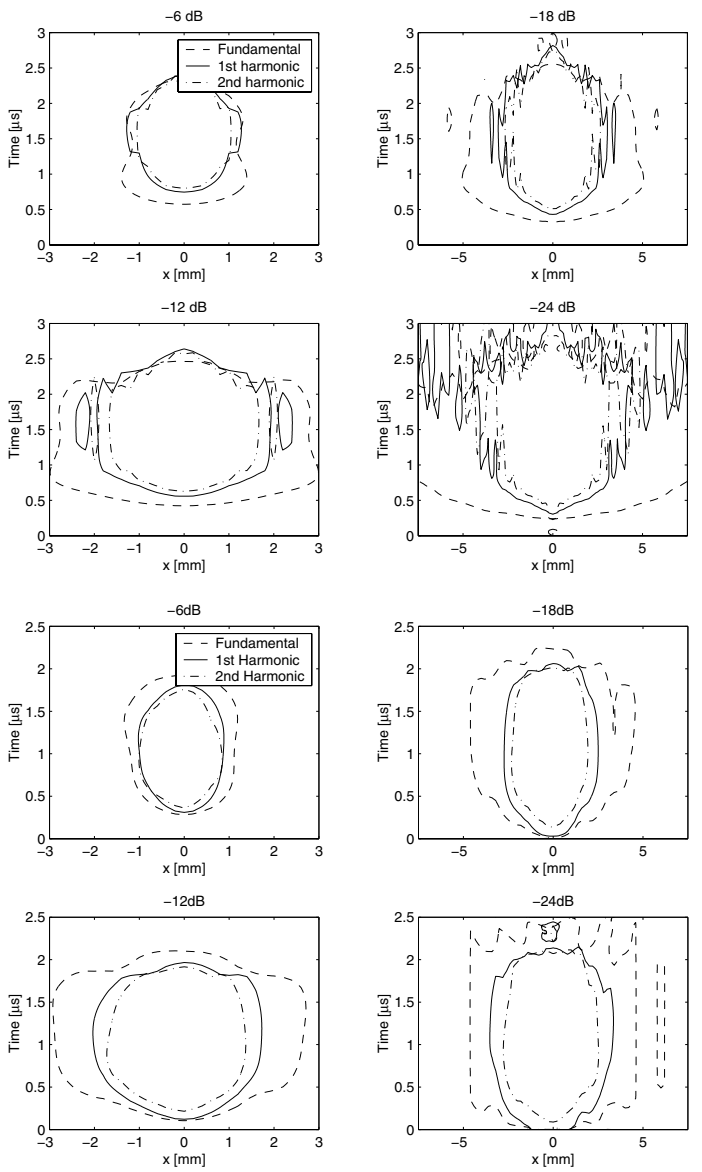

Figure 3: Simulated (top) and experimental (bottom) point spread functions obtained at a distance of $z=24 \mathrm{~mm}$.

using a collection of point scatterers as described in [12]. Their scattering strength is adjusted according to the physical structure of the object, and this can be used to simulate both cyst phantoms and real anatomic structures.

\section{ACKNOWLEDGEMENT}

This work was supported by grant 9700883, 9700563 and 5013-00-0025 from the Danish Science Foundation and by B-K Medical A/S and grant EF 918 from the Academy of Technical Sciences of Denmark.

\section{REFERENCES}

[1] J. A. Jensen and N. B. Svendsen. Calculation of pressure fields from arbitrarily shaped, apodized, and excited ultrasound transducers. IEEE Trans. Ultrason., Ferroelec., Freq. Contr., 39:262-267, 1992.

[2] J. Tavakkoli, D. Cathignol, and R. Souchon. Modeling of pulsed finite-amplitude focused sound beams in time domain. J. Acoust. Soc. Am., 104:2061-2072, 1998.
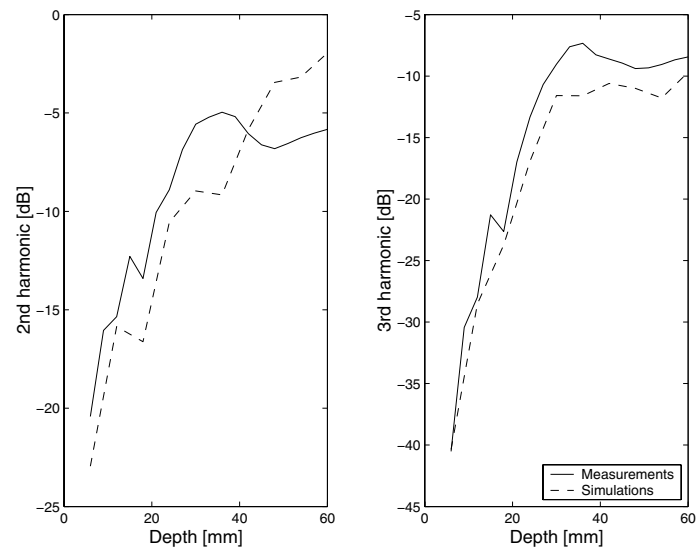

Figure 4: Simulated and experimental build-up of harmonic component as a function of depth. The seconds harmonic relative to the fundamental is shown on the left and the third harmonic on the right.

[3] J. P. Remenieras, O. Bou Matar, V. Labat, and F. Patat. Time-domain modeling of nonlinear distortion of pulsed finite amplitude sound beams. Ultrasonics, 38:305-311, 2000.

[4] L. K. Taylor, M. Schlaikjer, and J. A. Jensen. Modeling of higher harmonics formation in medical ultrasound systems. In Proc. of SPIE: Progress in biomedical optics and imaging, page To be published, 2002.

[5] P. J. Westervelt. Parametric acoustic array. J. Acoust. Soc. Am., 35:535-537, 1963.

[6] M. F. Hamilton and D. T. Blackstock. Nonlinear Acoustics. Academic Press, 1998.

[7] M. J. Haney and W. D. O'Brien. Temperature dependency of ultrasonic propagation properties in biological materials. In J. F. Greenleaf, editor, Tissue Characterization with Ultrasound. CRC Press, Boca Raton, Fla., 1986.

[8] G. E. Tupholme. Generation of acoustic pulses by baffled plane pistons. Mathematika, 16:209-224, 1969.

[9] P. R. Stepanishen. The time-dependent force and radiation impedance on a piston in a rigid infinite planar baffle. J. Acoust. Soc. Am., 49:841-849, 1971.

[10] J. A. Jensen, D. Gandhi, and W. D. O'Brien. Ultrasound fields in an attenuating medium. In Proc. IEEE Ultrason. Symp., pages 943-946, 1993.

[11] J. M. Burgers. A mathematical model illustrating the theory of turbulence. In R. von Mises and T. von Kármán, editors, Advances in Applied Mechanics, volume 1, pages 171-199. Academic Press, 1948.

[12] J. A. Jensen and P. Munk. Computer phantoms for simulating ultrasound B-mode and CFM images. In S. Lees and L. A. Ferrari, editors, Acoustical Imaging, volume 23, pages 75-80, 1997. 